Preprint typeset using $\mathrm{LAT}_{\mathrm{E}} \mathrm{X}$ style emulateapj v. 11/10/09

\title{
CHEMICAL TAGGING OF THREE DISTINCT POPULATIONS OF RED GIANTS IN THE GLOBULAR CLUSTER NGC $6752^{1}$
}

\author{
E. Carretta ${ }^{2}$, A. Bragaglia ${ }^{2}$, R.G. Gratton ${ }^{3}$, S. Lucatello ${ }^{3}$, V. D'Orazi ${ }^{4}$
}

\begin{abstract}
We present aluminium, magnesium, and silicon abundances in the metal-poor globular cluster NGC 6752 for a sample of more than 130 red giants with homogeneous oxygen and sodium abundances. We find that $[\mathrm{Al} / \mathrm{Fe}]$ shows a spread of about 1.4 dex among giants in NGC 6752 and is anticorrelated with $[\mathrm{Mg} / \mathrm{Fe}]$ and $[\mathrm{O} / \mathrm{Fe}]$ and correlated with $[\mathrm{Na} / \mathrm{Fe}]$ and $[\mathrm{Si} / \mathrm{Fe}]$. These relations are not continuous in nature, but the distribution of stars is clearly clustered around three distinct $\mathrm{Al}$ values, low, intermediate, and high. These three groups nicely correspond to the three distinct sequences previously detected using Strömgren photometry along the red giant branch. These two independent findings strongly indicate the existence of three distinct stellar populations in NGC 6752. Comparing the abundances of $\mathrm{O}$ and $\mathrm{Mg}$, we find that the population with intermediate chemical abundances cannot originate from material with the same composition of the most $\mathrm{O}$ - and $\mathrm{Mg}$-poor population, diluted by material with that of the most O- and Mg-rich one. This calls for different polluters.

Subject headings: Globular clusters: general — Globular clusters: individual (NGC 6752) — Stars: abundances — Stars: evolution — Stars: Population II
\end{abstract}

\section{INTRODUCTION}

Efficient multi-object high-resolution spectrographs allowed in recent years a major step forward in understanding multiple stellar populations in globular clusters (GCs). The large star-to-star abundance variations of light elements (C, N, O, F, Mg, Al, Si) were found to reflect the composition of at least two distinct stellar generations (e.g., Gratton et al. 2001 and the reviews by Gratton. Sneden \& Carretta 2004, Gratton, Carretta \& Bragaglia 2012). The huge gain in statistics not only provides a striking improvement for individual clusters, but better data available for large samples in a large number of GCs allow to distinguish cluster-to-cluster differences in the extension and shape of the related anticorrelations.

A good fraction of the observed stars comes from our ongoing FLAMES survey (Carretta et al. 2006, 2009a, b for a description), devoted to study the Na-O anticorrelation and its relation to global parameters in a large sample of GCs. Quantifying its extension, we were for instance able to show its dependence on the total absolute magnitude and metallicity of the clusters (Carretta et al. 2010) as well as its correlation with helium abundance (Gratton et al. 2010).

Better insight on the (still debated) nature of candidate polluters can be obtained by coupling the $\mathrm{NeNa}$ and the $\mathrm{MgAl}$ cycles. A good test-bed comes again from our FLAMES survey, and consists in more than 200 stars with FLAMES-UVES spectra, from which we derived simultaneously $\mathrm{O}, \mathrm{Na}, \mathrm{Mg}$, and $\mathrm{Al}$ abundances

\footnotetext{
${ }^{1}$ Based on data collected at the ESO telescopes under programme 085.D-0205

${ }^{2}$ INAF, Osservatorio Astronomico di Bologna, via Ranzani 1, 40127, Bologna, Italy. eugenio.carretta@oabo.inaf.it, angela.bragaglia@oabo.inaf.it

${ }^{3}$ INAF, Osservatorio Astronomico di Padova, vicolo dell'Osservatorio 5, 35122 Padova, Italy. raffaele.gratton@oapd.inaf.it sara.lucatello@oapd.inaf.it

${ }^{4}$ Dept. of Physics and Astronomy, Macquarie University, Sydney, NSW, 2109 Australia. valentina.dorazi@mq.edu.au
}

(Carretta et al. 2009b). The results show how in some clusters (usually massive and/or metal-poor, such as NGC 2808, NGC 6752) large amount of Al -and likely He- are produced, with efficient destruction of $\mathrm{O}$ and $\mathrm{Mg}$, whereas in others (e.g. M 4) no fresh $\mathrm{He}$ and $\mathrm{Al}$ is present. The major drawback is the limited sample available for each cluster (up to 14 stars observed with UVES Red Arm). To overcome this problem, we observed some interesting GCs with FLAMES-GIRAFFE to add $\mathrm{Al}$ abundances to the large set of $\mathrm{O}, \mathrm{Na}$, and $\mathrm{Mg}$ abundances already in our hand.

These observations may furthermore shed some light on the open issue of the continuous vs discrete nature of multiple stellar generations in GCs. At present, only in the case of the discrete main sequences in NGC 2808 the chemical signature of the $\mathrm{Mg}-\mathrm{Al}$ anticorrelation was found, directly studying a He-rich and a He-poor dwarf with X-shooter (Bragaglia et al. 2010) at intermediate resolution. In the other case of clearly separated main sequences, $\omega$ Cen, it was only possible to infer a very He-enriched distinct sequence using stacked spectra of similar resolution (Piotto et al. 2007). High resolution spectra of a large sample of stars (e.g.Marino et al. 2008 in M 4, with UVES) offer a better window on possible clumped distributions along the $\mathrm{Na}-\mathrm{O}$ anticorrelation, but only at a large cost in observing time (about 30 hours with UVES versus about 30 minutes with GIRAFFE of Carretta et al. 2009a). Hence, we exploited the efficient GIRAFFE HR21 grating to get a deeper insight on these two aspects: what were the first generation polluters (either intermediate-mass AGB stars, Ventura et al. 2001, or fast rotating massive stars, FRMS, Decressin et al. 2007), and whether the bursts of star formation for the different stellar generations in GCs represented a continuum or were discrete in nature. In this Letter we present the first results for NGC $6752\left([\mathrm{Fe} / \mathrm{H}]=-1.55, M_{V}=\right.$ -7.73 , Carretta et al. 2010), showing that our chemical tagging strongly confirm that three distinct populations are clearly traceable along the red giant branch (RGB) 
in this metal-poor cluster for which Milone et al. (2010) only found some evidence of an intrinsic broadening of the main-sequence. We briefly discuss the implications that the compositions of the three populations have on our understanding of the nature of the polluters.

\section{DATA AND ANALYSIS}

The abundances of aluminium were derived from GIRAFFE spectra (obtained with FLAMES, Pasquini et al. 2002, mounted at the ESO VLT-UT2 telescope) with the high resolution grating HR21. This set-up is centred at $8757 \AA$ and the spectral resolution is $\mathrm{R}=17,300$ at the center of spectra. The pointing was made adopting the same fibre positioning used by Carretta et al. (2007) to observe NGC 6752 with HR11, which includes the $\mathrm{Na}$ doublet at 5682-5688 $\AA$ : this choice maximizes the number of stars with both $\mathrm{Na}$ and $\mathrm{Al}$ abundances availabl5. The observations were made on July 2010, with an exposure time of 2700 seconds, and reduced by the dedicated GIRAFFE pipeline. The resulting 1-D, wavelength-calibrated spectra were sky subtracted, and shifted to zero radial velocity. The $\mathrm{S} / \mathrm{N}$ values span a range from about 50 up to more than 500 , with a median value of 192 .

Equivalent widths $(E W \mathrm{~s})$ of the $\mathrm{Al}$ lines were measured with the ROSA package (Gratton 1988) and abundances were derived using the atmospheric parameters already determined for each star in Carretta et al. (2007). A check with spectrum synthesis confirmed the reliability of our measurements. We use here LTE abundances. Andrievsky et al. (2008) computed N-LTE corrections for $\mathrm{Al}$ for very metal-poor stars, considering also the doublet used here. From their Fig. 2 the maximun differential effect for stars with temperature and gravity ranges similar to ours is less than 0.2 dex for $[\mathrm{Fe} / \mathrm{H}]=-$ 2 , the highest metallicity they consider, and arguably less at the cluster metallicity; futhermore, there is no trend of $\mathrm{Al}$ abundances with temperature or gravity in our data. The Mg abundances rest on two to three high excitation lines measured in the spectral ranges of HR11 and HR13, while Si was obtained from several transitions in the spectral range 5645-6145 $\AA$ (details and atomic parameters can be found in Carretta et al. 2009b and Gratton et al. 2003, respectively). The uppermost left panel in Fig. 1 shows the comparison of our $[\mathrm{Al} / \mathrm{Fe}] \mathrm{ra}-$ tios derived from the 8772-8773 $\AA$ doublet with those obtained by Yong et al. (2005) using the weaker doublet of $\mathrm{Al}$ I at 6696-98 $\AA$ in UVES spectra with spectral resolution ranging from $\mathrm{R}=60,000$ to 110,000 and with $\mathrm{S} / \mathrm{N}$ ratios from 100 to 250 per pixel. The agreement is excellent: our abundances are on average only 0.01 dex lower than those from this other study, with an r.m.s. scatter of 0.14 dex (13 stars). Abundances of $\mathrm{Mg}, \mathrm{Al}$, and $\mathrm{Si}$ for individual stars are listed in Table 1. Typical star-to-star errors in abundance ratios, due to errors on the adopted atmospheric parameters and $E W$ measurements, were estimated as in Carretta et al. (2007); they are $0.07,0.10$ and 0.05 dex for $[\mathrm{Al} / \mathrm{Fe}],[\mathrm{Mg} / \mathrm{Fe}]$ and $[\mathrm{Si} / \mathrm{Fe}]$, respectively.

\footnotetext{
${ }^{5} \mathrm{O}$ abundances, obtained using the HR13 grating and a slightly different fibre configuration, are not available for all the program stars
}

\section{ALUMINUM, MAGNESIUM, AND SILICON}

In the middle and lower panels of Fig. 1 we show the relations between the abundances of $\mathrm{Al}$ and of other proton-capture elements $\mathrm{Na}, \mathrm{O}, \mathrm{Mg}$, and $\mathrm{Si}$ in NGC 6752. We observe large star-to-star variations in the $\mathrm{Al}$ content, with a range of about 1.4 dex. The stars with the minimum (i.e., primordial) $\mathrm{Al}$ abundance also show the whole pattern typical of core collapse SN nucleosynthesis only: $[\mathrm{Na} / \mathrm{Fe}] \sim-0.1$ dex, $[\mathrm{Mg} / \mathrm{Fe}]$ and $[\mathrm{Si} / \mathrm{Fe}] \sim+0.45$ dex, $[\mathrm{O} / \mathrm{Fe}] \sim+0.5$ dex. The stars with most extreme abundances reach values of $[\mathrm{Al} / \mathrm{Fe}]$ as large as 1.4-1.5 dex. This observation by itself is a severe challenge to models invoking AGB stars as first generation polluters, since the only models able to produce such extreme abundances of Al (Ventura \& D'Antona 2009) are those of metal-poor $([\mathrm{Fe} / \mathrm{H}] \sim-2.3)$, low-mass (3.0-3.5 $\left.M_{\odot}\right)$ AGBs. However, these models do not show a depletion, but rather a simultaneous overproduction of $\mathrm{O}$. Moreover, stars in this mass range should experience a prolonged phase of 3rd-dredge up, likely resulting in enhanced $\mathrm{C}$ and $s$-elements, which is not observed in NGC 6752 (James et al. 2004). On the other hand, models of $5 M_{\odot}$ stars at the metallicity of NGC 6752 do yield abundances $[\mathrm{Al} / \mathrm{Fe}] \sim 1.2$ dex, not very far away from the observed maximum, but they fail to reproduce the whole range of enhancements observed in $\mathrm{Na}$ (see Table 2 in Ventura \& D'Antona 2009). Finally, even the nucleosynthesis of rapidly-rotating massive stars cannot reproduce such high $\mathrm{Al}$ abundances (see Decressin et al. 2007).

As expected, $\mathrm{Al}$ is well correlated with elements that are enhanced by the action of the $\mathrm{Ne}-\mathrm{Na}$ (such as $\mathrm{Na}$ ) and $\mathrm{Mg}-\mathrm{Al}$ cycles and is anticorrelated with elements that are depleted in H-burning at high temperature (such as $\mathrm{O}$ and $\mathrm{Mg}$ ). Furthermore, with the present work we confirm that in NGC 6752 the reaction network within the polluters of the first generation exceeded the temperature of $\sim 65 \times 10^{6} \mathrm{~K}$, the threshold required to generate the leakage from the $\mathrm{MgAl}$ cycle on ${ }^{28} \mathrm{Si}$, first discovered by Yong et al. (2005) in NGC 6752. The resulting $\mathrm{Al}-\mathrm{Si}$ correlation was then confirmed by Carretta et al. (2009b) using a limited sample of stars with UVES spectra, and is visible also in the lower-right panel in Fig. 1 based on a much more extended sample. This correlation is typically observed in metal-poor and/or massive clusters, like NGC 2808 and NGC 7078 (M 15; see Carretta et al. 2009b), and is a clear cut signature for the action of a class of first generation polluters so massive to reach such high temperature in their interiors.

\section{THREE DISTINCT SEQUENCES ON THE RGB IN NGC 6752}

The most striking result visible in the present large dataset is however the clumpy appearance of the distributions displayed in Fig. 1 Three groups of stars appear to be clustered around $[\mathrm{Al} / \mathrm{Fe}]$ values of about $0.1,0.8$, and 1.2 dex, respectively. This division seems reminiscent of that into the primordial (first generation, $\mathrm{P}$ ) and intermediate (I) and extreme (E) components of second generation defined in Carretta et al. (2009a) using $\mathrm{Na}$ and $\mathrm{O}$ abundances. To better distinguish the three subgroups, we plot in Fig. [2 the distribution of the $[\mathrm{Al} / \mathrm{Mg}$ ] values for red giants in NGC 6752. This ratio actually maximizes the signal along the anticorrelation and al- 
lows us to nicely trace three distinct groups with gaps at $[\mathrm{Al} / \mathrm{Mg}] \sim 0.0$ dex and $[\mathrm{Al} / \mathrm{Mg}] \sim 0.65$ dex. In turn, these gaps correspond quite well to the breaks clearly visible in the $\mathrm{Al}-\mathrm{Mg}$ anticorrelation at $[\mathrm{Al} / \mathrm{Fe}]=0.4$ and $[\mathrm{Al} / \mathrm{Fe}] \sim 1.0$ dex, both at $[\mathrm{Mg} / \mathrm{Fe}] \sim 0.45$ dex.

We identify the first break as a well defined border at the end of the star formation of the first stellar generation in the cluster. The novelty is that the gap between the groups of second generation stars with intermediate and extreme composition is now clearer than what we see using the $\mathrm{Na}-\mathrm{O}$ anticorrelation in the same stars. In the large dataset by Carretta et al. (2007) there is not a clear distinction between these groups; on the other hand, in the combined sample shown by Yong et al. (2003) (their Fig.4) three groups stand out in the Al-Na plane.

How solid is our finding? We can check this by using a fully independent approach. In Carretta et al. (2011) we used Strömgren multiband photometry, combined with information from spectroscopy, to study the possible separation and the properties of multiple populations in GCs. We tried to find the best combination of filters to enhance the differences (due to $\mathrm{N}$ and $\mathrm{C}$ molecular bands) and defined a new index $\delta_{4}=(u-v)-(b-y)$. This index seems to be better suited than others (such as $c_{y}$ by Yong et al. 2008) at separating first and second generation GC stars in the whole metallicity range sampled by GCs (see Carretta et al. 2011 for details).

Anyway, both indexes are able to show distinct structures on the RGB of NGC 6752 , as demonstrated by Fig. 3. NGC 6752 displays a tri-modal distribution, well evident both in the $y_{0}, c_{y, 0}$ and in $y_{0}, \delta_{4}$ color-magnitude diagrams (CMD). The conclusion from this sample of hundreds of stars is that in NGC 6752 there have been three episodes of star formation with well mixed gas in each individual phase.

Taking advantage of our newly derived abundances of $\mathrm{Al}$ and $\mathrm{Mg}$ for about 130 giants, we are now in the position to check this inference. We have about 110 stars in common with the sample of giants of NGC 6752 with accurate Strömgren photometry. In Fig. 3 we indicate stars from the present analysis, dividing them in three different groups, according to their $\mathrm{Al}$ abundances: stars with $[\mathrm{Al} / \mathrm{Fe}]<0.4$ dex (blue filled symbols), stars with $0.4<[\mathrm{Al} / \mathrm{Fe}]<1.05$ (green symbols) and stars with $[\mathrm{Al} / \mathrm{Fe}]>1.05$ dex. We see that (apart from a few outliers, most probably attributable to uncertainties both in the abundances and in the photometry) the segregation is strikingly evident: the three structures traced by photometry along the RGB in NGC 6752 nicely correspond to first generation stars in a tight sequence to the left of the RGB and to second generation stars separated into two discrete groups with moderate and very high nuclear processing, as indicated by their $\mathrm{Al}$ abundances.

\section{A CLUSTER ANALYSIS}

To better characterize the three different populations we found in NGC 6752, we applied a cluster analysis to our data, like that in Gratton et al. (2011) for $\omega$ Cen, using the $k$-means algorithm (Steinhaus 1956; MacQueen 1967) as implemented in the $R$ statistical package ( $\mathrm{R}$ Development Core Team, 2011)6. We con-

\footnotetext{
6 http://www.R-project.org
}

sidered five parameters for each star: [Na/Fe], [Mg/Fe], $[\mathrm{Al} / \mathrm{Fe}],[\mathrm{Si} / \mathrm{Fe}]$, and the residuals $\Delta\left(\delta_{4}\right)$ of $\delta_{4}$ values with respect to a mean line drawn through the points for NGC6752 in the $\left(y, \delta_{4}\right)$ diagram. The parameters were weighted according to their internal errors. A complete set of these parameters is available for 71 stars: the cluster analysis was done on these and we looked for a solution with three groups. The final division is actually dominated by the $\mathrm{Al}$ abundances, which have the largest variation with respect to internal errors. We called the three groups P, I, and E, because they look similar to the subdivision in Carretta et al. (2009a). In this sample, 15 stars are classified P, $26 \mathrm{I}$, and $30 \mathrm{E}$. We will not discuss here these ratios, but rather focus on the average abundance we obtain for the three different groups, listed in Table 2.

The three groups order consistently according to all parameters. Perhaps the most remarkable issue concerns the $\mathrm{Mg}$ and $\mathrm{O}$ abundances. If the I population simply formed by a fraction $d$ if the material of the E population diluted by a fraction $(1-d)$ of the material from the $\mathrm{P}$ population, the following relation should hold:

$$
d=\frac{\left[A(\mathrm{Mg})_{I}-A(\mathrm{Mg})_{P}\right]}{\left[A(\mathrm{Mg})_{E}-A(\mathrm{Mg})_{P}\right]}=\frac{\left[A(\mathrm{O})_{I}-A(\mathrm{O})_{P}\right]}{\left[A(\mathrm{O})_{E}-A(\mathrm{O})_{P}\right]}
$$

Instead, we get two very different values of $d$ from $\mathrm{O}$ and $\mathrm{Mg}(0.73 \pm 0.13 \mathrm{vs}$. $0.20 \pm 0.18)$. This fact is indeed confirmed by a simple look at the relation between $[\mathrm{O} / \mathrm{Fe}]$ and $[\mathrm{Mg} / \mathrm{Fe}]$ (see Fig. 2), which is quite obviously not linear.

We conclude that the material from which the I population formed was not produced by diluting material of the $\mathrm{E}$ population with material from the $\mathrm{P}$ population. $\mathrm{Mg}$ is not depleted in the I population as much as expected from this assumption, if the dilution is derived from $\mathrm{O}$. The conclusion is that the polluters for the $\mathrm{E}$ and I populations were different, the first one requiring H-burning at higher temperature than the second.

\section{DISCUSSION AND CONCLUSIONS}

In the present work we were able to provide a chemical tagging of three distinct sequences of stars along the RGB in NGC 6752, using the largest set of abundances of $\mathrm{O}, \mathrm{Na}, \mathrm{Mg}, \mathrm{Al}$, and $\mathrm{Si}$ for giants in this cluster.

The formation scenario and the nature of the polluters are different if the abundances can be described by a dilution model (see e.g. Prantzos and Charbonnel 2006) rather than by a number of discrete populations, each characterized by a given chemical composition. We examine our set of abundances from this point of view.

We provide two independent proofs that the distribution of giants in NGC 6752 is multi-modal, with stars segregated into three distinct groups, according to both Strömgren photometry and high-resolution spectroscopy. All these observations are based on the different composition presented by RGB stars. The large statistics from the photometry and the precise chemical tagging from our abundance analysis allow to reliably conclude that the groups showing a different chemistry are actually distinct.

As for the nature of the observed discreteness, it is easy to identify the first separation with the gap in star formation occurring at very early times between first and second generation stars. 
The second gap, observed among second generation stars, opens some questions. The definition of I and $\mathrm{E}$ groups of second generation stars, introduced in Carretta et al. (2009a) from $\mathrm{Na}, \mathrm{O}$ abundances (at $[\mathrm{O} / \mathrm{Na}]=-0.9 \mathrm{dex}$ ), is not entirely arbitrary, resting on the long tails observed in $[\mathrm{O} / \mathrm{Na}]$ ratios in some clusters like NGC 2808 or M 5, and returns fractions of I and E stars that nicely correlates with physical global parameters of GCs, like the total mass. In the case of NGC 6752, this neat separation, not immediately evident from the $\mathrm{Na}-\mathrm{O}$ anticorrelation, is quite clear when $\mathrm{Al}$ abundances are considered.

These results tell us that in NGC 6752 the interplay between star formation and dilution with pristine material acted so that two groups of stars with rather homogeneous chemical composition stand out. Thus, any mechanism resulting in discrete clumping must include an interruption either in the star formation or in the inflow of diluting material.

In addition, comparing $\mathrm{O}$ and $\mathrm{Mg}$ abundances we found that the composition of the I population cannot be obtained by diluting the material from which the E population was formed with those from which the $\mathrm{P}$ population originated. This calls for different polluters for the I and E populations.

This can be obtained in various ways. In the case of AGB stars we may think of two star formation episodes, separating the outcome of more massive and less massive AGB stars. An analogous scenario for FRMS is quite contrived, given their short lifetimes.

Another possibility is that both FRMS and AGB do contribute, at different epochs, to modify the primordial composition of stars in a GC, as suggested by Valcarce \& Catelan (2011). Were this the case, our observations require that the beginning of secondary star formation did not take place in the low mass tail of first generation stars: there must be a hiatus, albeit limited in time, between the two, else we should observe only two groups, not three.

Hence, we could envisage that the first contributors were FRMS that, owing to their very large masses, started to pollute the intracluster environment in the first few millions years after the burst of primordial star formation. After all the remaining gas has been either transformed into stars or blown away by SNe, no relevant amount of polluted matter was released with sufficiently slow velocity to be retained in the cluster. Only after about 50-100 Myr, when stars with an initial mass of about 7-5 $M_{\odot}$ entered the AGB phase, another injection of nuclearly processed matter occurred. Were the surface density of the accumulated gas above a certain threshold, star formation could be restarted forming stars with a chemistry of second generation clearly distinct from the progeny of FRMS.

This is only a qualitative sketch that, however, might account with sake of hypothesis for the existence of three separated populations of stars along the RGB in NGC 6752, although our data do not allow to attribute each individual group to a given class of polluters. This analysis might be applied to more metal-rich clusters. However, in these cases a careful examination of the possible contamination from $\mathrm{CN}$ features (scarcely relevant in the metal-poor cluster NGC 6752) must be included.

Partial funding comes from the PRIN INAF 2009 grant CRA 1.06.12.10 (PI: R. Gratton).

\section{REFERENCES}

Andrievsky, S. M., Spite, M., Korotin, S. A., et al. 2008, A\&A, 481,481

Bragaglia, A., Carretta, E., Gratton, R.G. et al. 2010, ApJ, 720, L41

Carretta, E., Bragaglia, A., Gratton R.G., Leone, F.,

Recio-Blanco, A., Lucatello, S. 2006, A\&A, 450, 523

Carretta, E., Bragaglia, A., Gratton, R.G., Lucatello, S. Momany, Y. 2007, A\&A, 464, 927

Carretta, E. et al. 2009a, A\&A, 505, 117

Carretta, E., Bragaglia, A., Gratton, R.G., Lucatello, S. 2009b, A\&A, 505, 139

Carretta, E., Bragaglia, A., Gratton, R.G., Recio-Blanco, A., Lucatello, S., D'Orazi, V., Cassisi, S. 2010, A\&A, 516, 55

Carretta, E., Bragaglia, A., Gratton, R.G., D'Orazi, V., Lucatello, S. 2011, A\&A, 535, 121

Decressin, T., Meynet, G., Charbonnel C. Prantzos, N., Ekstrom, S. 2007, A\&A, 464, 1029

Gratton, R.G. 1988, Rome Obs. Preprint Ser., 29

Gratton, R.G. et al. 2001, A\&A, 369, 87

Gratton, R.G., Carretta, E., Claudi, R., Lucatello, S., \& Barbieri, M. 2003, A\&A, 404, 187

Gratton, R.G., Sneden, C., \& Carretta, E. 2004, ARA\&A, 42, 385

Gratton, R.G., Carretta, E., Bragaglia, A., \& Lucatello, S., D'Orazi, V. 2010, A\&A, 517, 81

Gratton, R.G., Johnson, C.I., Lucatello, S., D’Orazi, V., \& Pilachowski, C. 2011, A\&A, 534, 72

Gratton, R., Carretta, E., \& Bragaglia, A. 2012, A\&AR, in press, arXiv: 1201.6526

James, G. et al. 2004, A\&A, 414, 1071

MacQueen, J. B. 1967, Mathematical Statistics and Probability, University of California Press., 281.

Marino, A.F., Villanova, S., Piotto, G., Milone, A.P., Momany, Y., Bedin, L.R., Medling, A.M. 2008, A\&A, 490, 625

Milone et al. 2010, ApJ, 709, 1183

Pasquini, L. et al. 2002, The Messenger, 110, 1

Piotto, G. et al. 2007, ApJ, 661, L53

Prantzos, N., Charbonnel, C. 2006, A\&A, 458, 135

R Development Core Team, 2011, R: A language and environment for statistical computing, Vienna, Austria, ISBN 3-900051-07-0

Steinhaus, H. 1956, Bull. Acad. Polon. Sci. 4, 801

Valcarce, A. A. R., \& Catelan, M. 2011, A\&A, 533, A120

Ventura, P., D'Antona, F. 2009, A\&A, 499, 835

Ventura, P. D'Antona, F., Mazzitelli, I., \& Gratton, R. 2001, ApJ, 550, L65

Yong, D., Grundahl, F., Lambert, D.L., Nissen, P.E., Shetrone, M.D. 2003, A\&A, 402, 985

Yong, D., Grundahl, F., Nissen, P.E., Jensen, H.R., Lambert, D.L. 2005, A\&A, 438, 875

Yong, D., Grundahl, F., Johnson, J.A., Asplund, M. 2008, ApJ, 684,1159 
Table 1

$[\mathrm{Mg} / \mathrm{Fe}],[\mathrm{Al} / \mathrm{Fe}]$ and $[\mathrm{Si} / \mathrm{Fe}]$ ratios for red giants in NGC 6752 . Complete table available on line only.

\begin{tabular}{|c|c|c|c|c|c|c|c|c|c|c|c|}
\hline star & $\mathrm{nr}$ & {$[\mathrm{Mg} / \mathrm{Fe}]$} & $\sigma$ & $\mathrm{nr}$ & {$[\mathrm{Al} / \mathrm{Fe}]$} & $\sigma$ & $\mathrm{nr}$ & {$[\mathrm{Si} / \mathrm{Fe}]$} & $\sigma$ & flagAl & flagPop \\
\hline 2162 & 1 & 0.452 & & 2 & 1.204 & 0.106 & 8 & 0.494 & 0.182 & 1 & - \\
\hline 4602 & 1 & 0.154 & & 2 & 1.184 & 0.040 & 7 & 0.527 & 0.189 & 1 & $\mathrm{E}$ \\
\hline 4625 & 2 & 0.423 & 0.051 & 2 & -0.098 & 0.008 & 8 & 0.401 & 0.144 & 1 & $\mathrm{P}$ \\
\hline 4787 & 2 & 0.384 & 0.093 & 2 & 1.150 & 0.082 & 11 & 0.491 & 0.214 & 1 & $\mathrm{E}$ \\
\hline
\end{tabular}

a $\mathrm{nr}$ is the number of lines.

b $\sigma$ is the $r m s$ scatter of the mean.

c flagAl: 1 for detection and 0 for upper limits in $\mathrm{Al}$

d flagPop: P, I, or E as derived from the cluster analysis

Table 2

Average values of parameters for stars in the three populations of red giants in NGC 6752 .

\begin{tabular}{|c|c|c|c|c|c|c|c|}
\hline Pop. & $\mathrm{nr}$ & $\Delta\left(\delta_{4}\right)$ & {$[\mathrm{O} / \mathrm{Fe}]$} & {$[\mathrm{Na} / \mathrm{Fe}]$} & {$[\mathrm{Mg} / \mathrm{Fe}]$} & {$[\mathrm{Al} / \mathrm{Fe}]$} & {$[\mathrm{Si} / \mathrm{Fe}]$} \\
\hline $\begin{array}{l}\mathrm{P} \\
\mathrm{I} \\
\mathrm{E}\end{array}$ & $\begin{array}{l}15 \\
26 \\
30\end{array}$ & $\begin{array}{l}-0.048 \pm 0.006 \\
-0.002 \pm 0.003 \\
+0.027 \pm 0.003\end{array}$ & $\begin{array}{l}+0.54 \pm 0.04 \\
+0.25 \pm 0.04 \\
+0.06 \pm 0.04\end{array}$ & $\begin{array}{l}+0.01 \pm 0.03 \\
+0.36 \pm 0.03 \\
+0.62 \pm 0.02\end{array}$ & $\begin{array}{l}+0.45 \pm 0.02 \\
+0.42 \pm 0.01 \\
+0.31 \pm 0.01\end{array}$ & $\begin{array}{l}+0.11 \pm 0.04 \\
+0.74 \pm 0.04 \\
+1.21 \pm 0.02\end{array}$ & $\begin{array}{l}+0.51 \pm 0.01 \\
+0.48 \pm 0.01 \\
+0.43 \pm 0.01\end{array}$ \\
\hline
\end{tabular}



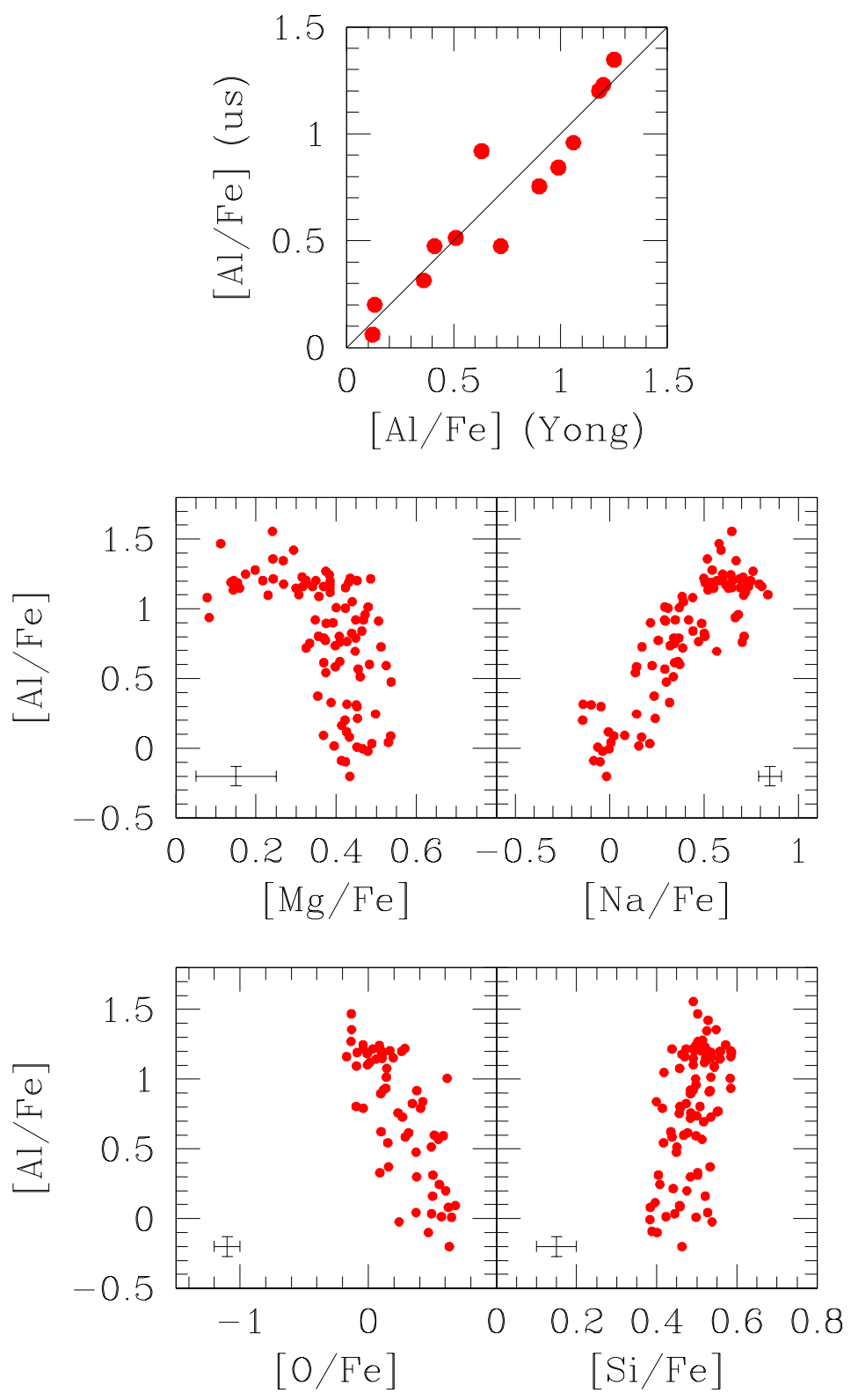

Figure 1. Upper panel, left: comparison of our $[\mathrm{Al} / \mathrm{Fe}]$ ratios with those from Yong et al. (2005) for 13 stars in common. The line of identity is indicated. Upper panel, right: the Mg-O correlation from our data (present work and Carretta et al. 2007). Middle panels: the Al-Mg anticorrelation (left) and the $\mathrm{Na}-\mathrm{Al}$ correlation (right). Lower panels: the same for the Al-O anticorrelation (left) and the Al-Si correlation (right). 


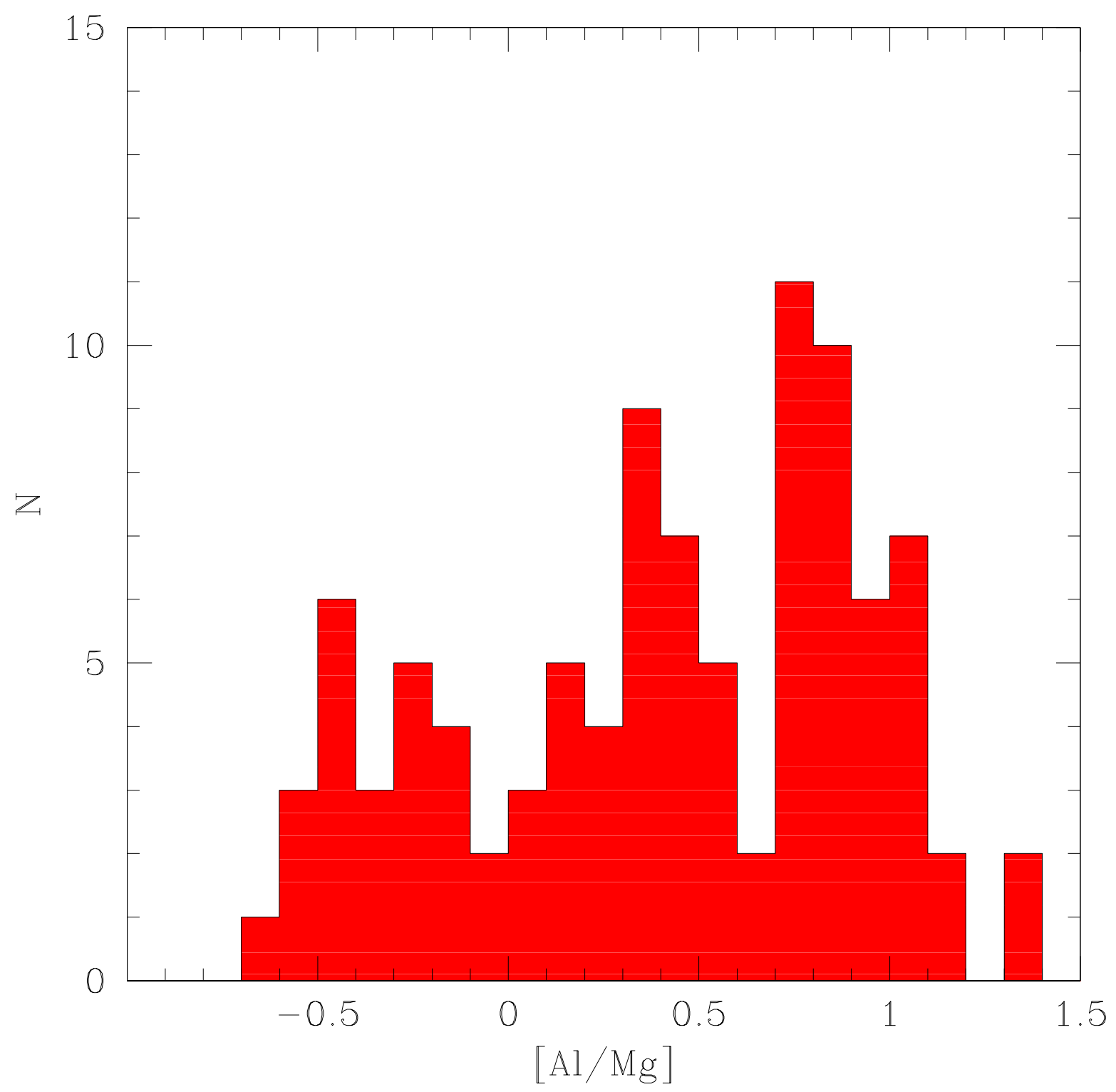

Figure 2. Distribution of the $[\mathrm{Al} / \mathrm{Mg}]$ ratios in red giants in NGC 6752 


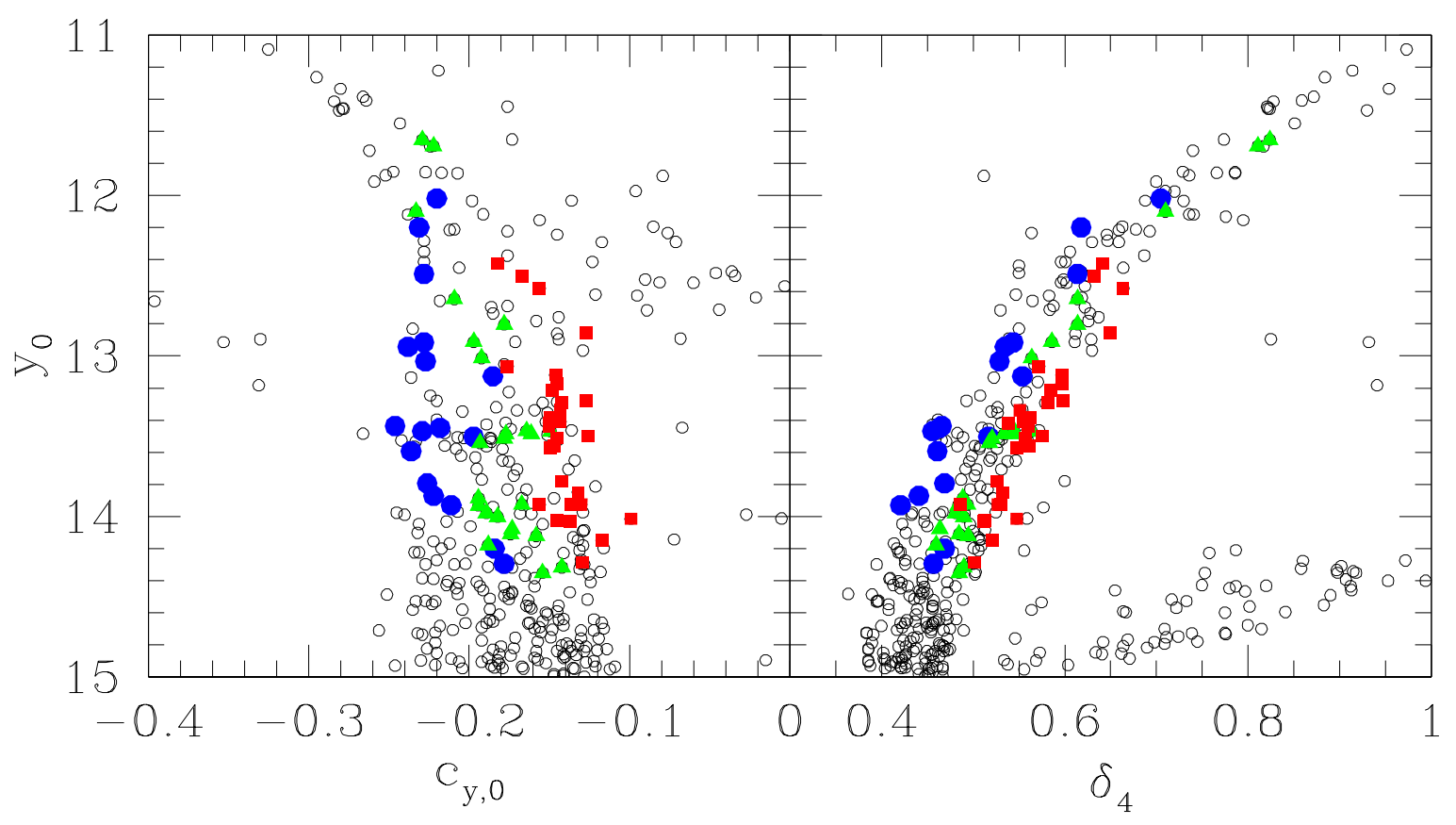

Figure 3. Left panel: Strömgren (dereddened) CMD in $y, c_{y}$, for NGC 6752 (empty grey circles). Superimposed in blue circles, green triangles and red squares are the stars with measured $\mathrm{Al}$ abundances: $[\mathrm{Al} / \mathrm{Fe}]<0.4$ dex, $0.4<[\mathrm{Al} / \mathrm{Fe}]<1.05 \mathrm{dex}$ and $[\mathrm{Al} / \mathrm{Fe}]>1.05$ dex, respectively. Right panel: the same for the CMD in $y, \delta_{4}$, where $\delta_{4}$ is a new index defined as $\delta_{4}=(u-v)-(b-y)($ see Carretta et al. 2011). 